\title{
Comparación de la biometría y metodología en el manejo de la Tortuga Golfina Lepidochelys olivacea (Olive ridley ) en Cedeño, Marcovia
}

Anny Murillo * ,Heber Contreras *, Guillermo Berrios *, Melissa Ramos *, Juan Carlos Carranza *, Roxy Betanco *, Yoleny Gómez *, Wilmer Hernández *, Vanessa Merlo **

\section{RESUMEN}

El propósito del estudio es realizar una comparación de la biometría y las metodologías empleadas en el monitoreo de la anidación de la Tortuga Golfina, en un periodo comprendido de 2010 - 2012. El área de estudio para este proyecto es en playa Edén, Cedeño, Marcovia del departamento de Choluteca, ubicada a $30 \mathrm{~km}$ noroeste de Choluteca. La extensión total de la playa es de una longitud de $8.5 \mathrm{~km}$, comprendida en los $13^{\circ} 08^{\prime} 08^{\prime \prime} \mathrm{N} 87^{\circ} 25^{\prime} 47^{\prime \prime} \mathrm{O}$. Área de influencia: Punta Ratón, El Venado, Boca de Río Viejo, considerado sitio preexistente de veda. La investigación se realiza mediante patrullajes nocturnos durante la época de veda, que se efectúa del 1 al 25 de septiembre de cada año. Esta acción de conservación y manejo se realiza de manera coordinada a través de la Comisión de Verificación y Control Ambiental del Golfo de Fonseca (CVC-GOLF).

Palabras Clave: avistamiento, desove, biometría, eclosión, patrullaje, especie bandera, transectos, veda, anidamiento.

\section{ABSTRACT}

The study's purpose is to compare the biometrics and methodologies employed in the monitoring of Olive Ridley nests in the period from 2010 to 2012. The project's area of study is in Eden beach located in Cedeño, Marcovia in the department of Choluteca, about $30 \mathrm{kms}$ northwest from Choluteca. The total length of the beach is $8.5 \mathrm{~km}$ long, between $13^{\circ} 08^{\prime} 08^{\prime \prime} \mathrm{N}$ and $87^{\circ} 25^{\prime} 47^{\prime \prime} \mathrm{W}$. The area of influence is Punta Raton, El Venado, Boca de Rio Viejo, which has been considered a pre existent closure site. The research has been done during night patrols in the closed season

Universidad Nacional Autónoma de Honduras. Centro Universitario Regional del Litoral Pacífico. Departamento de Acuicultura y Biología Marina. Carrera de Ingeniería en Ciencias Acuícolas y Recursos Marino Costeros.

Universidad Nacional Autónoma de Honduras. Centro Universitario Regional del Litoral Pacífico. Departamento de Acuicultura y Biología Marina. Carrera de Ingeniería en Ciencias Acuícolas y Recurso Marino Costero. Asesora.aramis_van28@yahoo.com 
time, which takes place from September 1 to September 25 each year. This conservation and management action is coordinated through the Verification Committee and Environmental Control of the Gulf of Fonseca. (CVC-GOLF).

Keywords: sighting, spawning, biometrics, hatching, patrol, flagship species, transects, nesting. 


\section{INTRODUCCIÓN}

Las tortugas marinas son base en el equilibrio del ecosistema marino, también representan el ingreso económico a muchas familias y actualmente han adquirido relevancia debido a el consumo desmedido, a las actividades turísticas, educativas, ambientales e investigativas que generan fuentes de empleo."Para conservar estos individuos y sus hábitats, deberán tomarse en cuenta extensiones amplias del planeta y manejarlas adecuadamente, y se convierte en un instrumento de protección para el mundo complejo e interconectado del que dependen las sociedades humanas". (Frazier, 2000)

Esta investigación se orienta a crear las condiciones de integración y empoderamiento por parte de los pobladores de Cedeño, para generar mejores condiciones ambientales y especialmente contribuir a la preservación y protección de los recursos naturales y específicamente de la Tortuga Golfina (Lepidochelys olivacea); la cual se encuentra en peligro de extinción. Será necesario el levantamiento de la información como línea de base que permitirá precisar, brindar validez y confianza en los datos obtenidos; además de fortalecer el trabajo que se desarrolla junto a la Comisión en la siembra de los huevos en la búsqueda del éxito de la eclosión de las tortugas. La Investigación realizada en la playa Edén durante el periodo de veda (01 al 25 de septiembre) servirá como aporte para recolectar información de soporte para conocer la densidad poblacional, factores implícitos de su biología y la conservación a estos quelonios que anidan en nuestras costas. Asimismo la comunidad a la cual apoyamos ha estado anuente a las actividades para su protección, como también aprovechando la oportunidad eco turística de toda la faja costera del pacífico hondureño. Los pobladores de la zona realizan esta actividad de manera artesanal. Esta investigación aportará información necesaria para que los pescadores cuenten con la información y el reconocimiento de las diferentes organizaciones que se encarguen de velar y conservar la Tortuga Golfina.

\section{MATERIALESY MÉTODOS}

\section{Área de estudio}

La zona seleccionada para este proyecto es la playa Edén, Cedeño, Marcovia del departamento de Choluteca, ubicada a $30 \mathrm{~km}$ Noroeste de Choluteca. Tiene una longitud de $8.5 \mathrm{~km}$, comprendida en los $13^{\circ} 08^{\prime} 08^{\prime \prime} \mathrm{N} 87^{\circ} 25^{\prime} 47^{\prime \prime} \mathrm{O}$. Área de influencia: Punta Ratón, El Venado y Boca de Río Viejo, considerando sitio preexistente de veda. Esta es una zona intensa de actividad pesquera y turística, lo que la considera un ecosistema marino frágil. El espacio marino comprende playas y estuarios que va desde playa Doradas hasta la comunidad de Delgaditos. 


\section{Sitios de patrullaje}

Durante este estudio se consideran 5 sitios de patrullaje:

1. Playa Cedeño Centro $\left(13^{\circ} 09^{\prime} 25.6^{\prime \prime} \mathrm{N} 87^{\circ} 25^{\prime} 46.1^{\prime \prime} \mathrm{W}\right)$, 2. Playa Edén $\left(13^{\circ} 08^{\prime} 36.4^{\prime \prime} \mathrm{N} 87^{\circ} 25^{\prime} 46.3 \mathrm{~W}\right), 3$. Estero Las Doradas desembocadura de estero

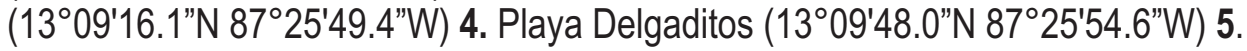
Playa de Cocos; para este sitio no se localizaron coordenadas sin embargo fue considerado en la tabulación de datos ubicados en los sitios antes indicados. Se realizaron rondas en las que observamos el arribo de las tortugas y apoyados de un GPS se encontraron las coordenadas de la anidación, una vez terminado el desove.

\section{Diseño}

Para este estudio se empleó el método comparativo y descriptivo de datos desarrollado en dos fases, en los días 24 y 25 de septiembre. Mediante la observación y basados en el avistamiento se tomaron las coordenadas, así mismo en esta fase se realizó el patrullaje de playa, esperando la anidación y la utilización de un instrumento GPS, para confirmación de las coordenadas. Una vez que la tortuga concluyó el desove se recolectaron los datos de biometría de tortuga, huevos y nidos. Seguidamente se procedió a la medición de la tortuga como: el ancho curvo del caparazón (ACC) y largo curvo del caparazón (LCC), ambas fueron tomadas con una cinta métrica. Las longitudes lineales del caparazón no fueron tomadas porque no se contaba con el instrumento necesario para realizarlo (pie de rey). Posteriormente se midió el peso promedio de los huevos utilizando una balanza digital y se contabilizó la cantidad de huevos por nido, al momento de ser extraídos. La manipulación de los huevos se realizó de manera prolija, recolectados en un recipiente plástico limpio; de la misma manera que se encontraban en el nido original y trasladándolos hacia el vivero artificial. Una vez evacuado el total de los huevos se mide el ancho y profundidad del nido.Seguidamente se observó cómo se elabora un vivero artificial, imitando principalmente la profundidad del nido original, procediendo de inmediato a la siembra de los huevos como estaban en el nido original y cubriéndolos con arena. Se procede a la rotulación del nido con la siguiente información: descripción del área de estudio, ubicación geográfica, extensión de la línea de playa, datos de la comunidad, número de nido (número correlativo), cantidad de huevos, hora de avistamiento, fecha y hora de la siembra, lugar del desove.

Finalmente, se empleó el método de transectos, para estimar el número de tortugas de acuerdo a la siguiente ecuación: $\check{N}=\left({ }^{n} \quad / 2 w / g\right) ~ A$, en donde $n=$ el número de avistamientos de la tortuga, $\mathrm{l}=$ la longitud del transecto, $\mathrm{w}=$ ancho del transecto al lado de la línea de rastreo, $\mathrm{g}=$ población de tortugas visibles, y $\mathrm{A}=$ el tamaño del área 
de estudio. La diferencia fundamental entre transectos de franja es que en el último la anchura w es escogido de manera simple, mientras en un transecto lineal w es estimado de los datos y se llama "ancho de banda efectivo". La variación de Ň se estima de la varianza de las réplicas del transecto lineal.

\section{RESULTADOS}

\section{ASPECTOS ECOLÓGICOS}

Datos recolectados durante la práctica de campo correspondiente a los días 24 y 25 de Septiembre de 2010

\begin{tabular}{|c|c|c|c|c|c|c|c|c|c|c|c|}
\hline 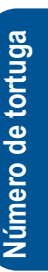 & $\begin{array}{l}\frac{\text { I }}{0} \\
\text { प } \\
4\end{array}$ & $\begin{array}{l}\text { 융 뜰 } \\
\text { 뜜융 } \\
\text { 옹 }\end{array}$ & 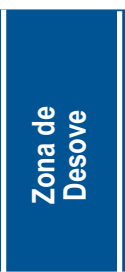 & 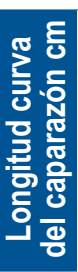 & 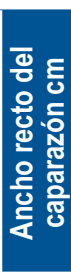 & 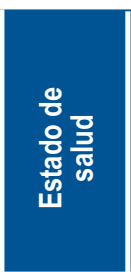 & 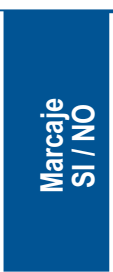 & 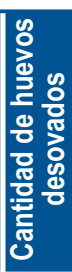 & 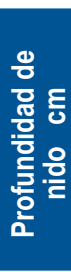 & $\begin{array}{l}\text { 홍 퉁 } \\
\text { 응 응 } \\
\frac{1}{4} \text { 은 }\end{array}$ & 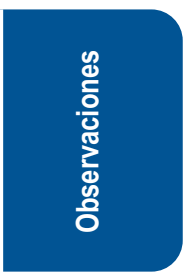 \\
\hline 1 & $24 / 09 / 2010$ & 07:15 pm & Doradas & 60 & 55 & Aceptable & SI 1728 & 110 & 27 & 19 & \\
\hline 2 & $24 / 09 / 2010$ & 09:15 pm & Edén & 61 & 42 & Aceptable* & NO & 104 & 38 & 21 & $\begin{array}{l}\text { *Manchas en } \\
\text { caparazón }\end{array}$ \\
\hline 3 & $24 / 09 / 2010$ & $12: 40 \mathrm{pm}$ & Edén & 83 & 56 & Aceptable & NO & 88 & 25 & 12 & \\
\hline 4 & $24 / 09 / 2010$ & $12: 34 \mathrm{pm}$ & Doradas & & & Aceptable & & 102 & 31 & 28 & $\begin{array}{l}\text { No se midió ya } \\
\text { que no se encon- } \\
\text { tró en la zona }\end{array}$ \\
\hline 5 & $24 / 09 / 2010$ & 01:30 pm & Delgaditos & & & Aceptable & & 86 & 47 & 29 & $\begin{array}{l}\text { No se midió ya } \\
\text { que no se encon- } \\
\text { tró en la zona }\end{array}$ \\
\hline 6 & $25 / 09 / 2010$ & 05:58 pm & Cedeño Centro & 55 & 35 & Aceptable & NO & 92 & 24 & 10 & \\
\hline 7 & $25 / 09 / 2010$ & 07:15 pm & Delgaditos & 55 & 32 & Aceptable & NO & 68 & 21 & 10 & \\
\hline 8 & $25 / 09 / 2010$ & 08:11 pm & Delgaditos & 55 & 32 & Aceptable & NO & 96 & 21 & 10 & \\
\hline 9 & $25 / 09 / 2010$ & $06: 45 \mathrm{pm}$ & Doradas & 39 & 68 & Aceptable & NO & 96 & 27 & 18 & \\
\hline 10 & $25 / 09 / 2010$ & 07:55 pm & Doradas & 37 & 70 & Aceptable & NO & 44 & 38 & 21 & \\
\hline 11 & $25 / 09 / 2010$ & 08:08 pm & Doradas & & & & & 94 & 25 & 12 & $\begin{array}{l}\text { No se midió ya } \\
\text { que no se encon- } \\
\text { tró en la zona }\end{array}$ \\
\hline 12 & $25 / 09 / 2010$ & $07: 20 \mathrm{pm}$ & Edén & 67 & 70 & Aceptable & NO & 118 & 40 & 18 & \\
\hline 13 & $25 / 09 / 2010$ & $08: 40 \mathrm{pm}$ & Edén & 64 & 44 & Aceptable & NO & 96 & 35 & 18 & \\
\hline 14 & $25 / 09 / 2010$ & 09:00 pm & Delgaditos & & & & & 108 & 39 & 23 & $\begin{array}{l}\text { No se midió ya } \\
\text { que no se encon- } \\
\text { tró en la zona }\end{array}$ \\
\hline 15 & $25 / 09 / 2010$ & 09:30 pm & Delgaditos & & & & & 67 & 21 & 12 & $\begin{array}{l}\text { No se midió ya } \\
\text { que no se encon- } \\
\text { tró en la zona }\end{array}$ \\
\hline
\end{tabular}

Durante las actividades se observaron un total de 15 tortugas de las cuales se completaron los datos de biometría de 10 tortugas avistadas, así como el peso promedio de huevos (4gr). Los datos faltantes de algunos individuos es porque se encontraron únicamente los rastros y los nidos durante el patrullaje. Todas las playas vigiladas durante la visita de campo tuvieron arribadas similares que 
corresponden 5 para Delgaditos, 5 Doradas, 4 Edén y solo 1 en el séctor de Cedeño Centro.

El día 24 de septiembre hubo un total de 5 tortugas avistadas y 10 para el día 25 , siendo este último con más arribadas avistadas.

Para la aplicación de la fórmula de estimación de población mediante el estudio de transectos se tomaron las siguientes medidas: ancho del transecto tomando la distancia entre la línea de playa en pleamar y el sitio de anidación, considerando el valor mayor:

$$
\begin{array}{lll}
1 & =30 \text { metros } & 5=30 \text { metros } \\
2 & =21 \text { metros } & 6=24 \text { metros } \\
3 & =27 \text { metros } & 7=38 \text { metros } \\
4 & =31 \text { metros } &
\end{array}
$$

La longitud del transecto se estimó la distancia del nido 1 al nido 6: del 1 al $6=$ $28.50 \mathrm{mts}$

El tamaño del área en estudio corresponde a toda la extensión de playa de $8.5 \mathrm{Km}$. (850 metros)

total de tortugas avistadas durante las fechas del estudio fueron 15 que corresponden a los días 24 y 25 de la veda, de las cuales solo se observaron 10 respectivamente. Realizando la aplicación de la fórmula determinamos:

$\check{\mathrm{N}}=(15$ individuos $/ 2$ (38mts. $28.5 \mathrm{mts} .10$ individuos $)) 850 \mathrm{mts}$ $\grave{\mathrm{N}}=0.58$ individuos/metro. Lo que significa que podemos encontrar 1 individuo cada 2 metros y para efectos de estimación de población se tomó la medida de la línea de playa y la longitud comprendida entre la línea de costa pleamar y punto de avistamiento de la tortuga hembra.

\section{DISCUSIONES}

Con respecto a los datos de tortugas arribadas por día, en el campamento en estudio los días más predominantes fueron el 1 y el 25 de la veda. La media y la moda indica que se necesita mayor Investigación ya que puede existir factores ambientales y antropogénicos que estén alterando estos resultados. 
El número de huevos desovados por tortuga indica que esta dentro del rango de acuerdo a las bibliografías citadas y hasta la fecha el porcentaje de éxito de eclosión en la playa Edén ha sido satisfactorio, aunque en varios nidos de los que han eclosionado existiendo presencia de hongos, es importante investigar los causales como la ubicación correcta del vivero o las fuertes lluvias que pudieron afectar.

La población estimada de individuos por extensión de playa es de 425. Se debe ampliar el tiempo del estudio para considerar datos biométricos y que se lleve de manera conjunta el registro de otras variables ambientales como ser: mareas, fases lunares, temperaturas y condiciones climáticas, esto para discutir los resultados investigados. Mantener las poblaciones existentes ayudará a sostener el equilibrio biológico; aunque se desconoce la cantidad de individuos diferenciados por sexo. No obstante esta información se desconoce por falta de equipo: termómetro de suelo, pluviómetro, termómetro ambiental y un barómetro para obtener la temperatura de la arena, ambiental, la precipitación pluvial, la humedad relativa y la presión atmosférica.

Un factor determinante para realizar un estudio son los datos recolectados. Es necesario que las personas encargadas estén debidamente capacitadas, por lo que se recomienda dar un seguimiento previo a la veda, entrenando a los miembros de la comunidad.

El campamento de playa Edén es un punto georreferencial estratégico al combinarse el agua costera con la del estero la convierte rica en nutrientes y abundante vegetación lo que se considera el mejor ecosistema para la reproducción y desarrollo de especies acuáticas.

Los datos obtenidos para realizar el estudio comparativo no están disponibles en los años 2007,2008, 2009 y 2010, para darle una secuencia. Esta información año con año ha ido mejorando sin embargo se requiere eficiente disponibilidad para efectos de estudio. Aunque el año 2005 únicamente se considera el campamento de Punta Ratón en comparación con el 2002 que están incluidos todos, refleja que hay una creciente en la cantidad de nidos. En el 2005 el porcentaje positivo de eclosión no fue satisfactorio, se tendría que evaluar que aspectos provocaron esta situación. Los datos del 2006 y 2010 no se han completado, pero el campamento de playa Edén representa un $23 \%$ del total nidos.

\section{CONCLUSIONES}

- Lepidochelys olivácea es la especie de tortuga marina más común en la costa sur de Honduras. Esta especie es un componente crítico en el sistema económico de 
la comunidad, debido a que la cosecha de huevos de estas especies está ligado a la economía de estas comunidades por lo tanto es necesario el entrenamiento de los pobladores de las comunidades que los llevaría a la dependencia sostenida de este recurso que es sumamente necesario.

- Fortalecer los campamentos con la presencia de equipos técnicos de las universidades, para que acompañen el proceso y permita organizar, sistematizar la información obtenida durante los periodos de veda; mediante equipo y capacitaciones -técnicas continuas, a los pobladores (comités de ambiente locales), con el fin de estandarizar métodos y mejorar el desarrollo de la labor de conservación.

- Ampliar el periodo de veda, considerando el consenso de los pobladores de las comunidades que residen en las zonas de anidamiento. Durante el proceso de levantamiento de información, se deberá contar con el equipo adecuado que permita obtener resultados confiables. (biometría)

- Debe formularse un proyecto socio-ambiental, en el que se incorporen líneas de acción orientadas al fortalecimiento de proyectos de subsistencia económica por parte de los pobladores de las comunidades de anidamiento en la zona sur, orientado a fortalecer esfuerzos de conservación del recurso, con el empoderamiento por parte de las comunidades en coordinación y comunicación de las autoridades de la junta directiva CVCGolfo de Fonseca y los comité de ambiente locales.

\section{BIBLIOGRAFÍA}

- Bolongaro Crevenna Recaséns, A., A. Z. Márquez García, V. Torres Rodríguez y A. García Vicario, 2010. Vulnerabilidad de sitios de anidación de tortugas marinas por efectos de erosión costera en el estado de Campeche, p. 73-96. En: A.V. Botello, S. Villanueva-Fragoso, J. Gutiérrez, y J.L. Rojas Galaviz (ed.). Vulnerabilidad de las zonas costeras mexicanas ante el cambio climático. Semarnat-ine, unam-ICMYL, Universidad Autonoma de Campeche.514 p

- Chacon, D.; Sanchez, J.; Calvo, J. y J. Ash.2007. Manual para el manejo y la conservación de las tortugas marinas en Costa Rica; con énfasis en la operación de proyectos en playa y viveros. Sistema Nacional de Áreas de Conservación (SINAC), Ministerio de Ambiente y Energía (MINAE). Gobierno de Costa Rica. San Jose.103.p

- Chacón, D.; Dick, B.; Harrison E.; .Sarti, L. y M. Solano. (2008). Manual sobre Técnicas de Manejo y Conservación de las Tortugas Marinas en Playas de Anidación de Centroamérica viveros. Sistema nacional de áreas de conservación (SIGNA), ministerio de ambiente y energía (MINAE) Gobierno de Costa Rica. San José 
- Dumbar, S. G., Salinas, L., and Castellanos, S.2011. Activities of Protective Turtle Ecology Center For Training, Ourtreach, and Research.Inc. (ProtECTOR) in Punta Raton, Honduras. 2009-2010 Annual Report, Ministry of Environment, DIGEPESCA, Honduras.

- Desarrollo sostenible. (2012) Consultado el 22 de septiembre 2012. http://www.cinu.mx/temas/medio-ambiente

- Eckert, K. L., K.A.Bjondal, F:A. Abreu-Grobois y M. Donnelly (Editores). 2000 (Traducción al Español). Técnicas de investigación y Manejo para la Conservación de las Tortugas Marinas. Grupo Especialista en Tortugas marinas UICN/CSE. Publicación No.4.

- García-Romero, M.E.; Hernández-Dueñas, L.M; García-Castañeda, B.; Santos. Soto, A. y Meyer-Willerer, A.O. "Protección y conservación de tortugas marinas de la zona costera de Michoacán México. Red de Revistas Científicas de América Latina y el Caribe, España y Portugal. México. Avances en investigación agropecuaria. Vol. 11. Número 002. 2007. p15-22 